livraisons

d'Histoire

de l'Architecture

\section{Livraisons de l'histoire de l'architecture}

32 | 2016

Les représentations de l'architecture

\title{
La représentation de l'architecture dans les arts industriels, une historicisation des décors (1800-1830)
}

The representation of architecture in industrial art, tracing its development in the early $19^{\text {th }}$ century (1800-1820)

Die Architekturdarstellung im Kunstgewerbe, eine Historisierung des Dekors (1800-1820)

\section{Aziza Gril-Mariotte}

\section{OpenEdition}

\section{Journals}

Édition électronique

URL : http://journals.openedition.org/lha/639

DOI : $10.4000 /$ lha.639

ISSN : 1960-5994

Éditeur

Association Livraisons d'histoire de l'architecture - LHA

Édition imprimée

Date de publication : 31 décembre 2016

Pagination : 65-88

ISSN : 1627-4970

Référence électronique

Aziza Gril-Mariotte, «La représentation de l'architecture dans les arts industriels, une historicisation des décors (1800-1830)», Livraisons de l'histoire de l'architecture [En ligne], 32 | 2016, mis en ligne le 31 décembre 2018, consulté le 10 décembre 2020. URL : http://journals.openedition.org//ha/639 ; DOI : https://doi.org/10.4000//ha.639 


\section{LA REPRÉSENTATION DE L'ARCHITECTURE DANS LES ARTS INDUSTRIELS, UNE HISTORICISATION DES DÉCORS (1800-1830)}

Dans la première moitié du XIX $\mathrm{X}^{\mathrm{e}}$ siècle, un engouement pour l'architecture s'empare du textile imprimé et des papiers peints panoramiques, les monuments deviennent un thème à la mode. Les toiles pour ameublement, imprimées à la plaque de cuivre, selon l'adaptation du procédé de l'estampe, se sont répandues en France depuis les années 1770, déployant une technique inventée en Angleterre ${ }^{1}$. $\mathrm{Au} \mathrm{XVIII}$ siècle, ces compositions se distinguent par la diversité de leurs thèmes où les motifs d'architecture sont fréquemment employés par les dessinateurs qui peuplent d'édifices les jardins et chinoiseries ou bien de formes pittoresques les compositions champêtres. Ces étoffes figuratives sont vendues sous le terme de "camaïeux à personnages " ou "meubles à personnages ", affirmant leur conception figurative et leur finalité décorative. Dans ces toiles, les représentations d'architectures imaginaires ou de monuments réels sont indissociables de l'esthétique de la ruine dont les décors se développent dans de nombreuses compositions selon la tradition de la peinture de genre et de paysage. Ces motifs forment un cadre pour des saynètes et restent le plus souvent limités à des ornements secondaires.

À partir de 1799, le vocabulaire ornemental est profondément renouvelé sous l'influence du néoclassicisme qui se manifeste dans des compositions ordonnées selon une conception décorative, abandonnant le principe des saynètes dans un paysage. Dans les années 1810, les manufactures de papier peint, Dufour à Paris et Zuber à Rixheim en Alsace se spécialisent dans la fabrication de panoramiques, assemblant plusieurs lés de papier pour constituer un paysage. Au XVIII siècle, les fabricants ont proposé des coordonnées papier-textile en reprenant des motifs

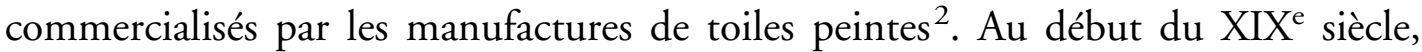
les industriels affirment leur indépendance esthétique avec le panoramique qui transforme les intérieurs, faisant du mur le support pour évoquer un ailleurs, plus

1. Peter Floud, English printed textiles, 1720-1836, Victoria \& Albert Museum, Londres, Her Majesty Stationery Office, 1960 ; Stanley Chapman, Serge Chassagne, European textile printers in the Eighteenth century, a study of Peel and Oberkampf, Londres, 1981.

2. Josette Bredif, "Étude des similitudes de motifs entre toiles imprimées et papiers peints de la seconde moitié du XVIII ${ }^{\mathrm{e}}$ siècle ", Bulletin du C.I.E.T.A., 1995-1996, nº 73, p. 106-116; "Étude des similitudes de motifs entre toiles imprimées et papiers peints de la seconde moitié du XVIII e siècle " Copier, coller, papiers peints $d u$ XVIII $I^{e}$ siècle, actes du colloque de Neuchâtel, 8 et 9 mars 1996, Neuchâtel, Département de l'instruction publique et des affaires culturelles, Service de la protection des monuments et des sites, 1998, p. 143-155. 
ou moins lointain, mais bien souvent réel. Si ces décors partagent, en partie, les thèmes décoratifs du textile imprimé, en particulier les représentations d'architectures, leur conception décorative s'en distingue nettement. Au moment où les meubles à personnages abandonnent le principe du paysage au profit d'une formule décorative, accentué par l'ornement du fond, l'industrie du papier peint propose avec les panoramiques une vision en trompe-l'œil dont la finalité est d'ouvrir le mur vers l'extérieur.

C'est dans ce contexte que la représentation de l'architecture se déploie sur de nouveaux supports ${ }^{3}$. Dans un premier temps, elle est visible dans les motifs liés à l'égyptomanie et plus largement dans les décors évoquant des contrées lointaines et exotiques, avant que les monuments forment de nouveaux sujets pour les compositions de meubles. Au XIX ${ }^{\mathrm{e}}$ siècle, la valeur accordée aux monuments va au-delà de l'intérêt grandissant pour les antiquités, cet engouement pour le passé historique aboutit à la représentation de véritables monuments. Les dessinateurs ne se contentent plus de quelques motifs, désormais, ils attribuent aux motifs architecturaux une importance nouvelle dans leurs compositions. Plus que le monument, c'est la place de l'architecture et les moyens graphiques mis en ouvre qui attestent d'une profonde évolution. Ce phénomène résulte-t-il d'un effet de mode ou bien d'un transfert entre différentes productions industrielles? Quelle est la valeur accordée à ces représentations et comment les choix iconographiques ont-ils participé à la construction d'une histoire monumentale? Quelles sont les solutions formelles imaginées par les dessinateurs pour mettre en œuvre ces motifs d'architectures sur des supports avec une fonction décorative? À partir d'un corpus varié, les choix des dessinateurs et des fabricants permettent de comprendre l'engouement du thème de l'architecture dans des productions d'arts industriels dont la finalité est de décorer les intérieurs.

\section{Que nous raconte l'architecture dans les toiles imprimées et le papier peint}

$\mathrm{Au}$ XVIII siècle, l'architecture se limite aux motifs de ruines ou aux fabriques qui peuplent les jardins à l'anglaise, les meubles à personnages sont alors pensés comme un paysage où des figures participent à des saynètes pittoresques ${ }^{4}$. La relation entre les figures et l'architecture reprend le principe du paysage en peinture en se propageant dans les motifs de pastorales, les décors d'architecture sont ainsi largement utilisés pour agrémenter le paysage. Dans la toile "ruines romaines " 5 ,

3. L'intérêt pour la représentation des monuments parisiens n'est pas nouveau comme le montre les tableaux d'Hubert Robert présentés au Salon de 1789 sur "les Monuments antiques de la France " et sur "des principaux Édifices de Paris ", voir Guillaume Faroult (dir.), Hubert Robert (17331808) un peintre visionnaire, catalogue de l'exposition au musée du Louvre, du 8 mars au 30 mai 2016, Paris, éd. Somogy, p. 386-387.

4. Aziza Gril-Mariotte, Les Toiles de Jouy. Histoire d'un art décoratif(1760-1821), Rennes, PUR, 2015, p. 168-170.

5. Ruines romaines, manufacture Petitpierre et $C^{\text {ie }}$, v. 1785, Paris, Musée des arts décoratifs, inv. E 18. 
commercialisée en 1785 par la manufacture Petitpierre et Cie à Nantes, plus que de simples ruines, le dessinateur met en scène des vestiges archéologiques, les motifs architecturaux sont identifiables. Il transpose l'esthétique de la ruine, chère aux peintres des vedute qui se passionnent pour les antiques de l'Italie, représentés dans des paysages peuplés d'admirateurs ou de simples paysans ${ }^{6}$. Cet exemple est précurseur d'une vision du monument comme modèle historique et artistique. Cette

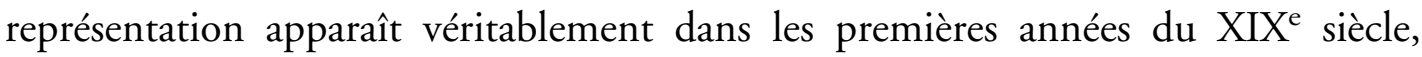
lorsque les manufacturiers répondent à l'engouement pour les contrées lointaines et les récits historiques, puis pour les grandes capitales culturelles. Alors qu'au $\mathrm{XVIII}^{\mathrm{e}}$ siècle le monument reste un élément de décor contribuant à la mise en scène pittoresque, désormais il répond au double impératif de situer les personnages dans un temps et dans un lieu. À la fin du XVIII siècle, à la manufacture de Jouy, Christophe-Philippe Oberkampf demande à son principal dessinateur de meubles, le peintre Jean-Baptiste Huet, d'adapter ses compositions à l'évolution du goût sous l'Empire. Pour se renouveler, il puise dans les modèles de la décoration des Loges de Raphaël au Vatican et dans les monuments antiques diffusés par la gravure ${ }^{7}$. Pour ce genre d'étoffes destinées à l'ameublement, le dessinateur propose plusieurs compositions qui se distinguent par la place accordée à l'architecture. Il semble être le premier à s'intéresser à la représentation de monuments qui se propage dans les manufactures de toiles peintes en Alsace et à Rouen et sur d'autres supports quand les fabriques de papier peint s'emparent à leur tour des vues de villes ${ }^{8}$.

Lorsque les manufactures proposent aux consommateurs de nouvelles compositions évoquant des pays lointains, l'architecture prend une place plus ou moins importante au regard des personnages. Après l'expédition d'Égypte et le retour triomphant de Bonaparte, alors que les arts décoratifs sont gagnés par l'égyptomanie, les dessinateurs transposent des images rapportées par les artistes où se mêlent vestiges archéologiques, paysage pittoresques et personnages orientaux. Dans le « meuble égyptien" (ill. 1), commercialisé en 1807 par la manufacture de Jouy, Jean-Baptiste Huet assemble des monuments de lieux et d'époques différents : le fort de Quaitbay dans le port d'Alexandrie, l'obélisque, dit de Cléopâtre, près de Louxor et des colonnes de temples pharaoniques. Ce mélange se reflète dans les autres motifs où des personnages orientaux sont disposés à côté de sculptures égyptiennes, d'entrées

6. Kees Boschma, "Quelques considérations sur les vedute au XVIII ${ }^{\mathrm{e}}$ siècle ", Le Paysage en Europe du $X V I^{e}$ au XVIII siècles, actes du colloque, Musée du Louvre, 25-27 juin 1990, Catherine LoiselLegrand, Jean-François Mejanes, Emmanuel Starcky (dir.), Paris, RMN, 1994, p. 151-169.

7. Recueil d'arabesques contenant les Loges du Vatican gravées d'après Raphä̈l d'Urbin par Pierre-Philippe Choffard, Paris, chez Chéreau, 1785-1790 ; Nicolas Ponce, Les Arabesques antiques des bains de Livie et de la Villa Adrienne avec les plafonds de la Villa Madame, peints d'après les dessins de Raphaël, Paris, 1789.

8. La manufacture Züber à Rixheim propose plusieurs papiers peints panoramiques comme Vues d'Italie, tandis que d'autres manufactures moins connues déclinent ce principe avec de grandes villes, Paris, Lyon. Voir Bernard Jacqué, De la manufacture au mur, pour une histoire matérielle du papier peint (1770-1914), thèse de doctorat d'histoire sous la direction de Serge Chassagne, Université de Lyon II Lumière, 2003. 


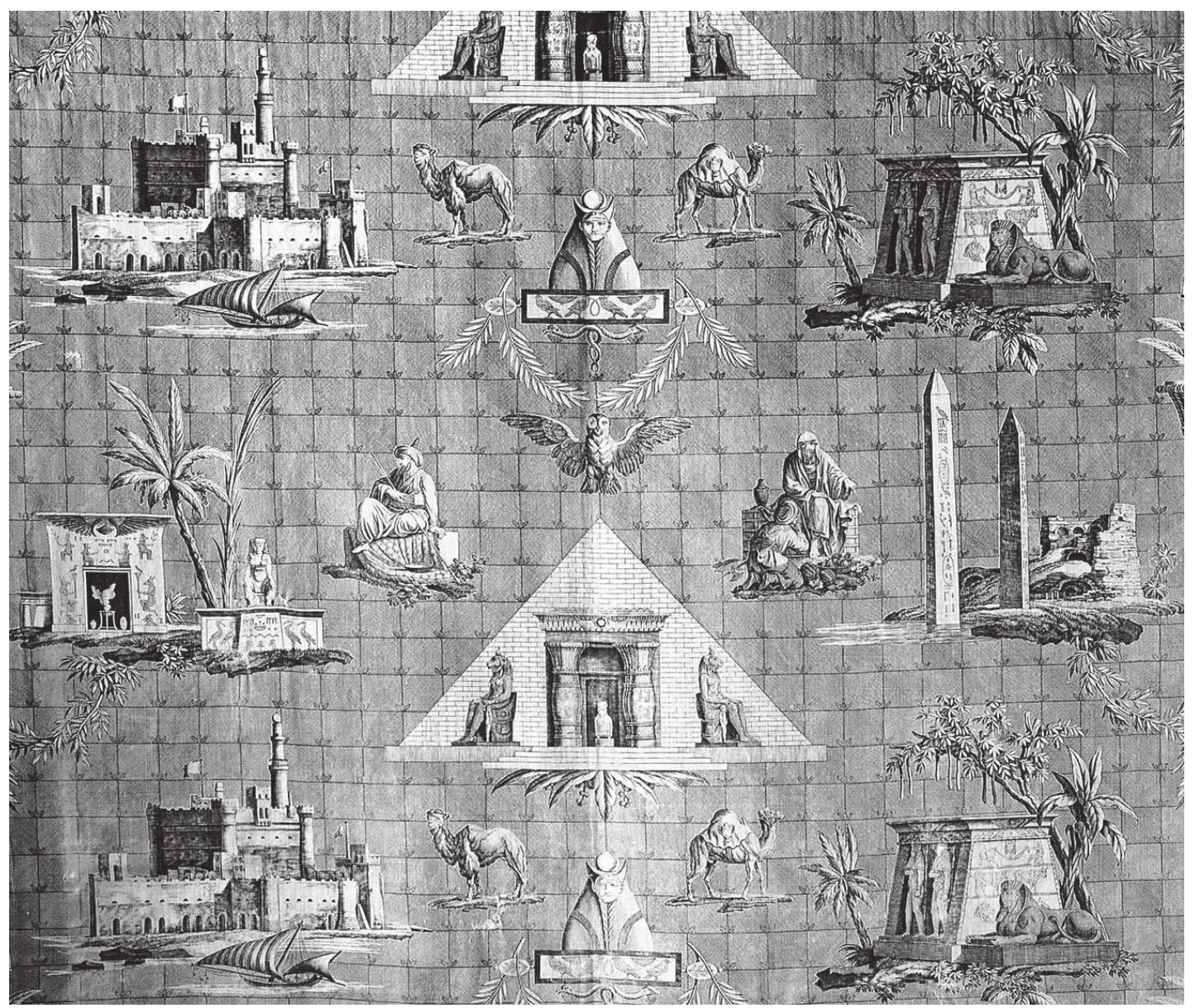

Ill. 1 : Les Monuments d'Égypte, dessinateur Jean-Baptiste Huet, impression à la plaque de cuivre, vendue sous le nom de «meuble égyptien », 1807, D326, rapport $52 \times 95 \mathrm{~cm}$. Musée de la toile de Jouy (MTJ) inv.982.44. (C) Cl. Marc Walter/Musée de la toile de Jouy.

de temple ou encore d'une statue de sphinx. La vision de l'Égypte reste celle d'un voyage pittoresque alors que les monuments apparaissent tels des estampes illustrant le récit d'un voyageur. Les saynètes sont pourtant juxtaposées selon un ordonnancement géométrique, issu de l'esthétique de l'arabesque dont les recueils diffusent des formules décoratives souvent simplifiées ${ }^{9}$. La représentation de l'architecture est associée au souvenir du voyage, mais le décoratif prime sur l'histoire avec les motifs se découpant sur l'appareillage des pyramides qui orne le fond de la toile.

9. Recueil d'arabesques contenant les Loges du Vatican gravée d'après Raphä̈l d'Urbin; et grand nombre d'autres compositions du même genre dans le style antique d'après Normand, Quéverdo, Salembier, Prieur, Boucher, Dugourc et d'autres, Paris, chez Joubert, 1802. Dans cet ouvrage destiné surtout aux décorateurs, Charles Normand reprend intégralement les décors de grotesques des pilastres gravés par Volpato, puis par Choffard avec les arabesques des Loges et de la Villa Madame, complétés par des compositions contemporaines dans le même style. Charles Normand, Nouveau recueil en divers genres d'ornements et autres objets propre à la décoration, tels que panneaux, vases, plafonds, candélabres, autels, trépieds, cassolettes, sarcophages, coupes, frises, camées, bas-reliefs, masques, lits, chaises, fauteuils, bergères, tables, bureaux, secrétaires, autres meubles, etc. composés, dessinés et gravés au trait, Paris, chez Joubert, 1803. 
Le dessinateur emprunte à l'architecture pharaonique la forme de la pyramide dont les motifs de briques se détachent en fond et mettent en valeur les vestiges archéologiques.

Les monuments symbolisent l'histoire et la culture d'un pays ; la représentation des paysages avec décors et ornements montre que l'artiste privilégie une vision pittoresque, tout en se référant au goût pour l'égyptomanie. Les monuments participent à la mise en scène de lieux décrits par les voyageurs et diffusés par des récits illustrés ${ }^{10}$. Le thème de la composition devient la découverte d'un pays, dépassant le contexte de l'égyptomanie ${ }^{11}$. Dans les années 1820, l'image de l'Égypte est reprise dans une manufacture rouennaise par le graveur Louis Henri Bréviaire (ill. 2) avec un meuble où les architectures ne sont plus que de simples décors agrémentant des saynètes. La vision décorative empruntée à l'arabesque chez JeanBaptiste Huet laisse place à une succession de saynètes, disposées sur le fond orné de la toile. Louis Henri Bréviaire s'empare du voyage en Orient selon une esthétique inspirée des peintres orientalistes, tout en conservant des vestiges archéologiques, mais dont la représentation est plus symbolique que réelle. On ne reconnaît aucun monument connu et surtout leur place dans le dessin se limite à quelques éléments dans le lointain alors que les personnages se déploient au premier plan dans des saynètes, évoquant le pittoresque de l'Orient. Le graveur s'inspire de scènes orientalistes et des cavaliers diffusés par l'estampe, d'après Horace et Carle Vernet $^{12}$. Mais plus que leur identification, c'est leur taille largement inférieure aux figures qui donne une autre signification à la composition. Entre 1807 et les années 1830, la représentation de l'architecture a laissé place à celle des habitants avec leurs costumes exotiques, suscitant l'intérêt des consommateurs européens.

Cette relation entre les habitants d'un pays et des monuments n'est pas nouvelle, elle a déjà été employée en 1810 par Jean-Baptiste Huet dans une composition sur le thème de la Chine ${ }^{13}$. Dans cette toile, l'architecture est intégrée à des paysages, les monuments accompagnant des scènes de la vie quotidienne. Le dessinateur privilégie une figuration du peuple chinois en déployant des saynètes où

10. La portée de l'expédition d'Égypte de Bonaparte est d'une importance considérable pour les arts grâce à la "commission des sciences et arts ", forte de 160 hommes qui participent à l'exploration scientifique du pays. Dès 1802, paraît l'ouvrage de Vivant Denon, Voyage dans la Basse et la HauteÉgyte pendant les campagnes du général Bonaparte, accompagné de nombreux dessins de l'auteur. Puis, à partir de 1809 paraît aux presses impériales, la monumentale Description de l'Égypte, véritable encyclopédie des connaissances acquises sur l'antiquité pharaonique et gréco-romaine, l'histoire naturelle et la société égyptienne de l'époque.

11. Jean-Marcel Humbert, L'Égyptomanie : sources, thèmes et symboles, étude de la réutilisation des thèmes décoratifs empruntés à l'Égypte ancienne dans l'art occidental du XVI siècle à nos jours, thèse de doctorat d'histoire de l'art sous la direction de Jean Leclant, Paris-IV Sorbonne, 1987 ; Égyptomanie (l') à l'épreuve de l'archéologie, actes du colloque international organisé au musée du Louvre, sous la direction scientifique de Jean-Marcel Humbert, 8 et 9 avril 1994.

12. Le Victoria \& Albert Museum de Londres possède une série de lithographie d'Horace Vernet de cavaliers orientaux qui ont sans doute inspiré le graveur.

13. Aziza Gril-Mariotte, Les Toiles de Jouy, op. cit., p. 239 : Les Chinois, dessinateur Jean-Baptiste Huet, impression à la plaque de cuivre, 1810, numéro de Dessin 383, rapport $52 \times 95 \mathrm{~cm}$. 


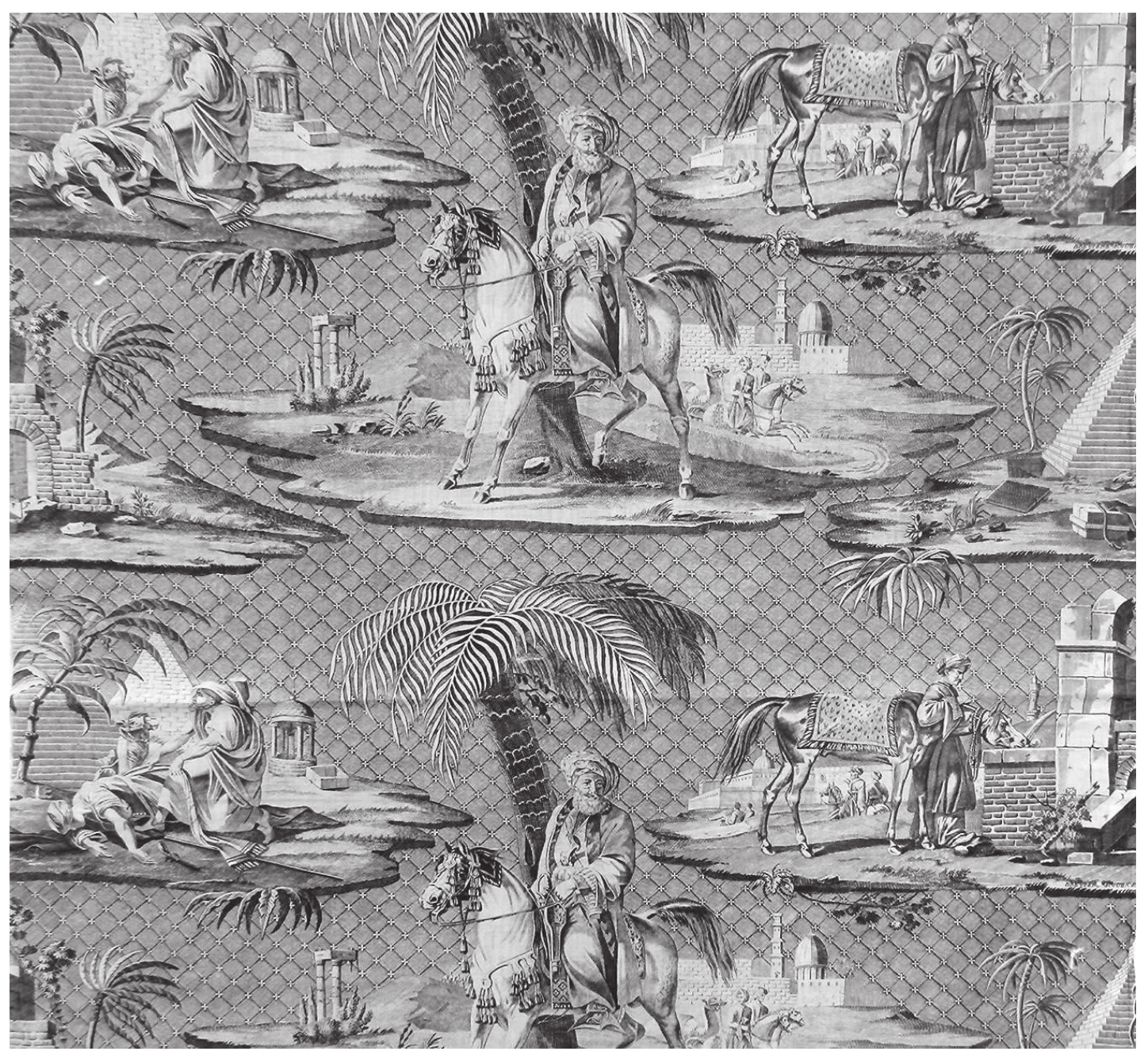

Ill. 2 : Scènes d'Égypte, manufacture rouennaise (signature Brévière, Rouen F), vers 1820, impression à la plaque de cuivre, $86 \times 123 \mathrm{~cm}$, rapport $47 \times 86 \mathrm{~cm}$. Musée de l'impression sur étoffes (MISE) inv.961.282.1.2. (C) Cl. de l'auteur.

les personnages sont identifiables à leurs costumes, plutôt qu'une représentation géographique du pays. Les monuments historiques accompagnent des scènes typiques, le dessinateur empruntant à des gravures des lieux connus : la forteresse de Yangzhou et le Potala de Lhassa ${ }^{14}$. Les édifices sont choisis afin de placer les figures dans un espace vraisemblable alors que la toile est vendue sous le titre commercial «Les Chinois». La manufacture propose aux consommateurs une nouvelle image de la Chine, loin des représentations exotiques et fantaisistes des chinoiseries du XVIII ${ }^{\mathrm{e}}$ siècle.

L'évocation d'un pays à travers ses monuments emblématiques, complétée par des paysages habités de paysans, de pêcheurs et de soldats chinois révèle une relation entre histoire et patrimoine culturel. La Chine est toujours une contrée lointaine, mais la multiplication des voyages et la publication de nouveaux récits 


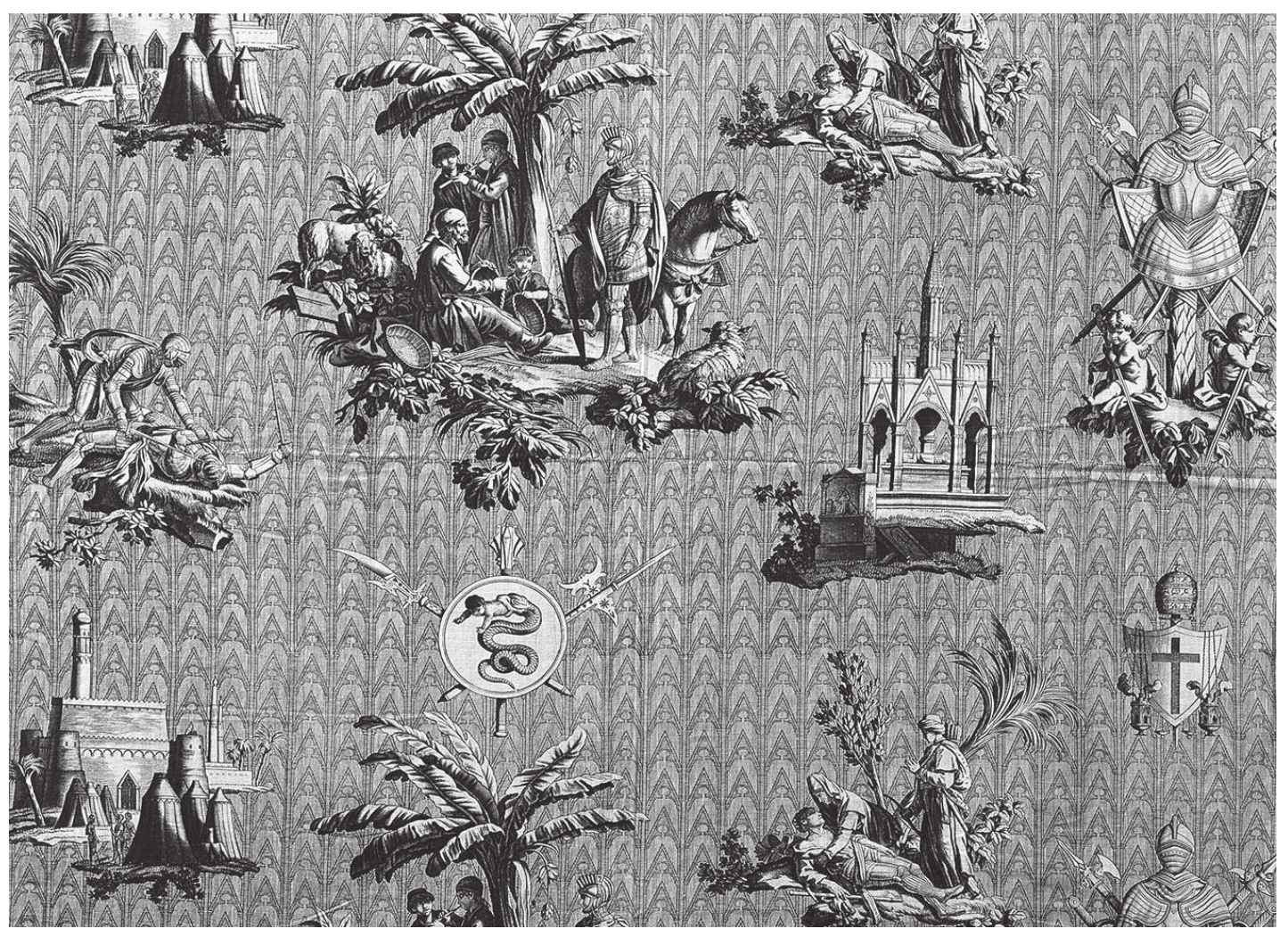

Ill. 3: Tancrède et Herminie, dessinateur Jean-Baptiste Huet, impression à la plaque de cuivre, 1809, D349, rapport $52 \times 94 \mathrm{~cm}$. MTJ inv.999.9.4. ( C Cl. Marc Walter/Musée de la toile de Jouy.

contribuent à faire connaître les réalités étrangères de ce pays ${ }^{15}$. L'architecture, réelle ou imaginaire, en Égypte ou en Chine, est devenue dans ces toiles d'ameublement un décor pour évoquer des horizons lointains. Ces exemples accordent une place importante aux motifs architecturaux dont les formes étranges suscitent la curiosité, mais la place accordée aux habitants, identifiables par leurs costumes traditionnels montre que les compositions proposent aux consommateurs un voyage imaginaire dans le temps et l'espace.

Lorsque le public se passionne pour les sujets empruntés au Moyen Âge, les dessinateurs ont également recours à l'architecture pour situer leurs dessins dans un temps et un espace géographique. En 1809, Jean-Baptiste Huet réalise une composition sur le thème de La Jérusalem délivrée (ill. 3), poème épique du Tasse, mis à la mode par l'opéra et le goût pour la littérature de la Renaissance. Les principaux motifs restent les scènes dans lesquelles se déroule l'intrigue entre Tancrède

15. Les Lettres édifiantes et curieuses de Chine (1702-1776) publiées par la mission jésuite ont contribué à faire connaître les mœurs de la Chine, tout en entretenant l'attrait pour l'exotisme. À la fin du $\mathrm{XVIII}^{\mathrm{e}}$ siècle, les Anglais sont les premiers à proposer des ouvrages illustrés qui font connaître les réalités géographiques et culturelles de la Chine : Sir Georges Staunton, An Authentic Account of on Embassy from the King of Great Britain to the Emperor of China, Londres, 1797 ; William Alexander, The Costume of China illustrated in forty-eight coloured engravings, Londres, 1805. 
et Herminie qui donne son titre à la toile ${ }^{16}$. Les monuments sont des éléments signifiants : la forteresse de Jérusalem sert de décor au camp des Croisés tandis que le monument funéraire gothique révèle l'influence du musée des Monuments français d'Alexandre Lenoir. La composition reprend le principe symétrique mis au point par le dessinateur dans le meuble égyptien, le tombeau gothique n'est alors qu'un motif parmi d'autres. Dans cet exemple, la représentation de l'architecture peut sembler anecdotique, la mise en scène de l'art gothique est l'élément décoratif mis en avant par Jean-Baptiste Huet. Il reprend l'idée du fond orné par un élément architectural avec des ogives placées en bandes verticales, transformant un élément du tombeau en une forme décorative démultipliée. La référence à l'architecture est devenue une source de création pour le dessinateur qui répond à la demande du fabricant de renouveler le style des compositions en s'affranchissant des paysages du XVIII ${ }^{e}$ siècle. À nouveau, l'architecture symbolise une contrée lointaine, pouvant être associée au voyage historique, tandis que le thème de Tancrède et Herminie est en accord avec le style troubadour à la mode au Salon dans les mêmes années ${ }^{17}$.

Dans le textile imprimé comme dans le papier peint, l'architecture est associée au voyage. Après la découverte de contrées lointaines, inaccessibles pour les consommateurs, les manufactures proposent des motifs plus familiers et proches avec la représentation de Paris. À partir des années 1810, les monuments de la capitale sont des motifs à la mode dans les arts industriels tandis que la peinture se passionne pour les représentations de panoramiques de ville ${ }^{18}$. En 1812, la manufacture Dufour semble avoir eu la première l'idée de composer un panoramique mettant en scène de véritable "portraits" de monuments parisiens (ill. 4) ${ }^{19}$. Le papier peint propose une promenade entre l'Hôtel de Ville, les églises du Val-deGrâce et de Saint-Sulpice, le Palais Bourbon, la fontaine du Châtelet devant la colonnade du Louvre et la colonne Vendôme. Cette conception de la représentation du monument comme un site à visiter est renforcée par la présence de promeneurs au premier plan de l'autre côté de la Seine. La disposition des personnages en dehors de l'espace où se trouvent les architectures évoque un dispositif théâtral, confortant la perception des monuments comme des ouvres à admirer.

16. Aziza Gril-Mariotte, Les Toiles de Jouy, op. cit., p. 222 : Tancrède et Herminie, dessinateur JeanBaptiste Huet, impression à la plaque de cuivre, 1809, numéro du Dessin 349, rapport $52 \times 94 \mathrm{~cm}$.

17. François Pupil, Le Style troubadour ou la nostalgie du bon vieux temps, Nancy, Presses universitaires de Nancy, 1985, p. 49.

18. Pierre Prévost, Panorama de Paris pris du toit du pavillon de Flore, v. 1805-1813, crayon et gouache sur papiers marouflés sur toile, 64 sur $817 \mathrm{~cm}$, Galerie Kugel. Je tiens à remercier Véronique de la Hougue, conservateur en chef du département des papiers peints au musée des Arts Décoratifs, de m'avoir signalé ce panorama. La mode des panoramas s'étend aux autres grandes capitales comme Rome, Henri Lebert dans son journal évoque lors d'un voyage à Paris sa visite à l'exposition «du panorama de Rome» en 1818 : Journal manuscrit autographe, vol. III (1816-1819), dépôt de la $\mathrm{BnF}$ à la bibliothèque municipale de Colmar.

19. Odile Nouvel-Kammerer (dir.), Papiers peints panoramiques, musée des Arts Décoratifs, Flammarion, 1998 , p. 276. 

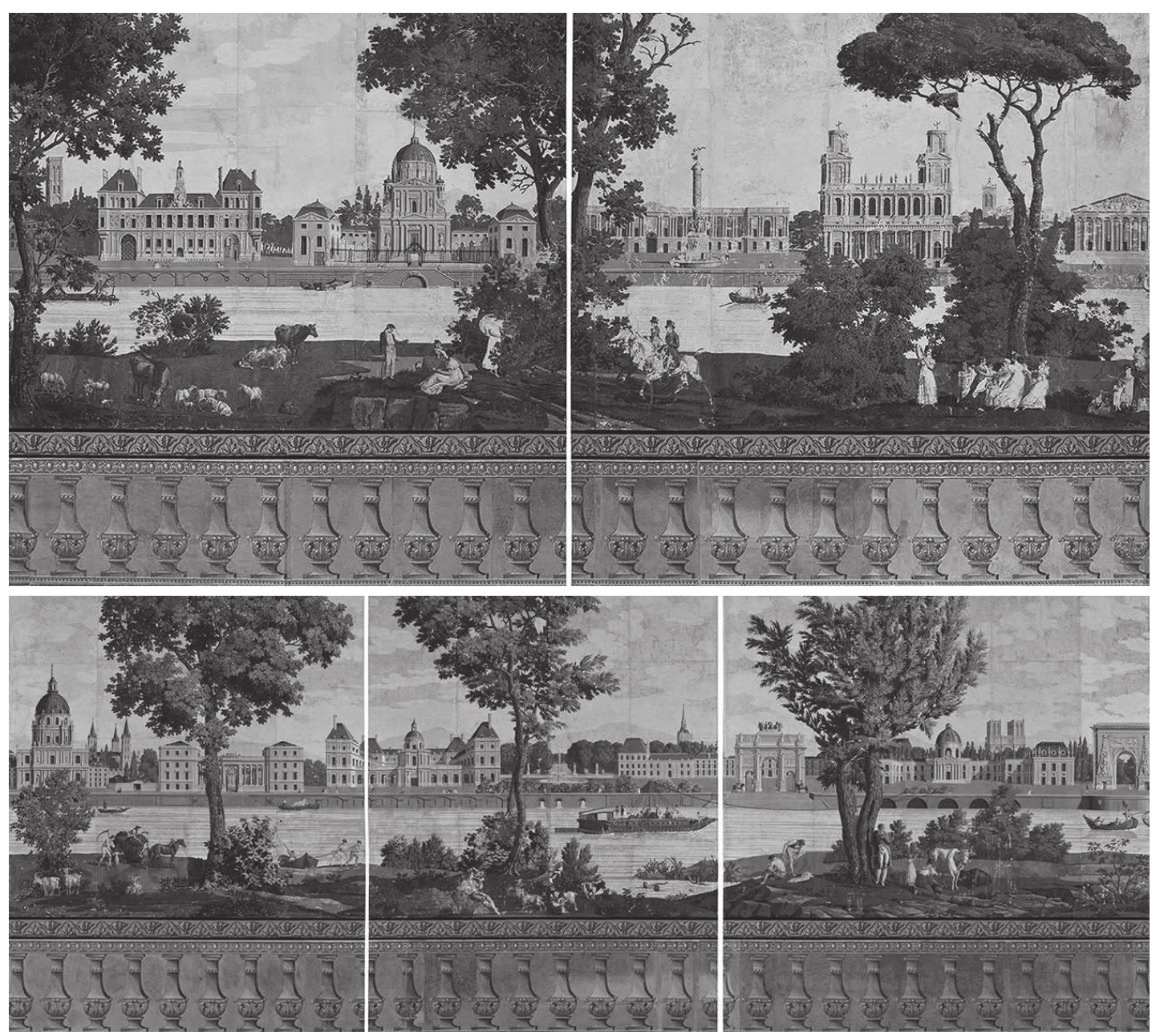

Ill. 4 : Les Monuments de Paris, papier peint panoramique de la manufacture Joseph Dufour, édition originale 1812-1815, réédition 1815-1830 avec suppression de la statue de Napoléon. Impression sur papier rabouté, dim. panneau $183 \times 193 \mathrm{~cm}$. L'Hôtel de Ville et le Val-de-Grâce, Saint-Sulpice et le Palais Bourbon, la fontaine du Châtelet devant la colonnade du Louvre. (C) Cl. Les Arts Décoratifs, Paris/Jean Tholance.

Plusieurs meubles imprimés suivront cette composition: la manufacture de Jouy commercialise en 1816 une toile dont le dessin (ill. 5) réalisé par l'architecte Louis-Hippolyte Lebas présente la colonnade du Louvre et Sainte-Geneviève, le Pont-Neuf et la fontaine des Innocents, comme des estampes illustrant un récit de voyage, mais en abandonnant l'aspect pittoresque des visiteurs. Au même moment en Alsace, la manufacture Hartmann \& Cie à Munster imprime une autre composition dans laquelle de nombreux monuments et architectures sont disposés sur le fond orné d'un fin quadrillage (ill. 6) ${ }^{20}$. Ce meuble propose un panorama de sites

20. Vies privées. Histoire singulière de l'impression textile, musée de l'Impression sur Étoffe de Mulhouse, catalogue de l'exposition du 7 octobre 2000 au 10 février 2002, p. 81-84. Le meuble est attribué à l'entreprise de commerce en toileries et indiennes Soëhnée l'Aîné \& $C^{\text {ie }}$ qui est associé à la manufacture d'André Hartmann père. Voir Louis Bergeron, Banquiers, négociants, manufacturiers parisiens du Directoire à l'Empire, Paris, 1978, chap. VII, "Le négoce parisien et son rôle dans le commerce français et international ", Paris, éd. EHESS, p. 167-204. 


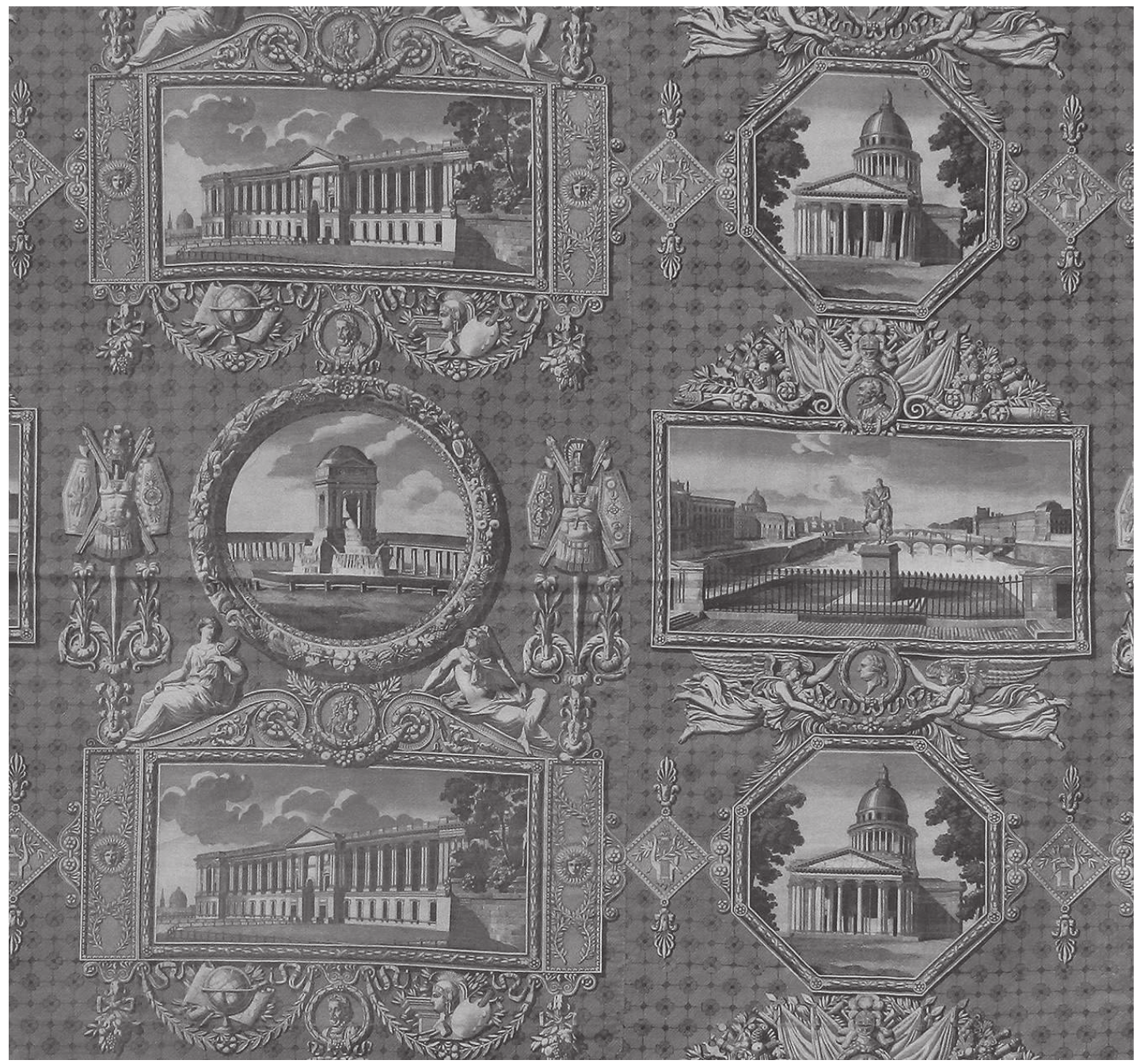

Ill. 5 : Les Monuments de Paris, dessinateur Louis-Hippolyte Lebas, 1816, D600, impression à la plaque de cuivre, rapport 52,5 $\times 82 \mathrm{~cm}$. MISE 961.605.1. ( C Cl. de l'auteur.

parisiens diffusant une image artistique et politique de la ville: le Palais des Tuileries, l'Arc de triomphe du Carroussel, la colonne Vendôme, les bâtiments de la place de la Concorde donnant sur la Madeleine, la porte Saint-Denis, les Invalides, le Palais Bourbon et l'église Saint-Sulpice. Cette succession de lieux fait écho aux voyages fréquents du fabricant à Paris, souvent accompagné de son dessinateur Henri Lebert, suivant les préconisations dans la formation des artistes pour l'industrie textile en vigueur depuis le XVIII siècle $^{21}$.

Le panoramique du fabricant Dufour comme la toile imprimée du manufacturier Hartmann exposent une vision de la capitale comme un lieu de prome-

21. Antoine-Nicolas Joubert de L'Hiberderie, Le dessinateur pour les fabriques d'étoffes d'or, d'argent et de soie, Paris, Sébastien Jorry, 1765, p. 88 : "Le fruit que peut tirer un dessinateur du voyage de Paris, c'est-à-dire, indication de tout ce qu'il y a de curieux relativement à la peinture $\&$ au dessein : comme les plus fameux magasins d'étoffes, cabinets d'estampes \& de peinture, particuliers \& publics, édifices, équipages, manufactures, maisons royales, statuaires, spectacles ». 


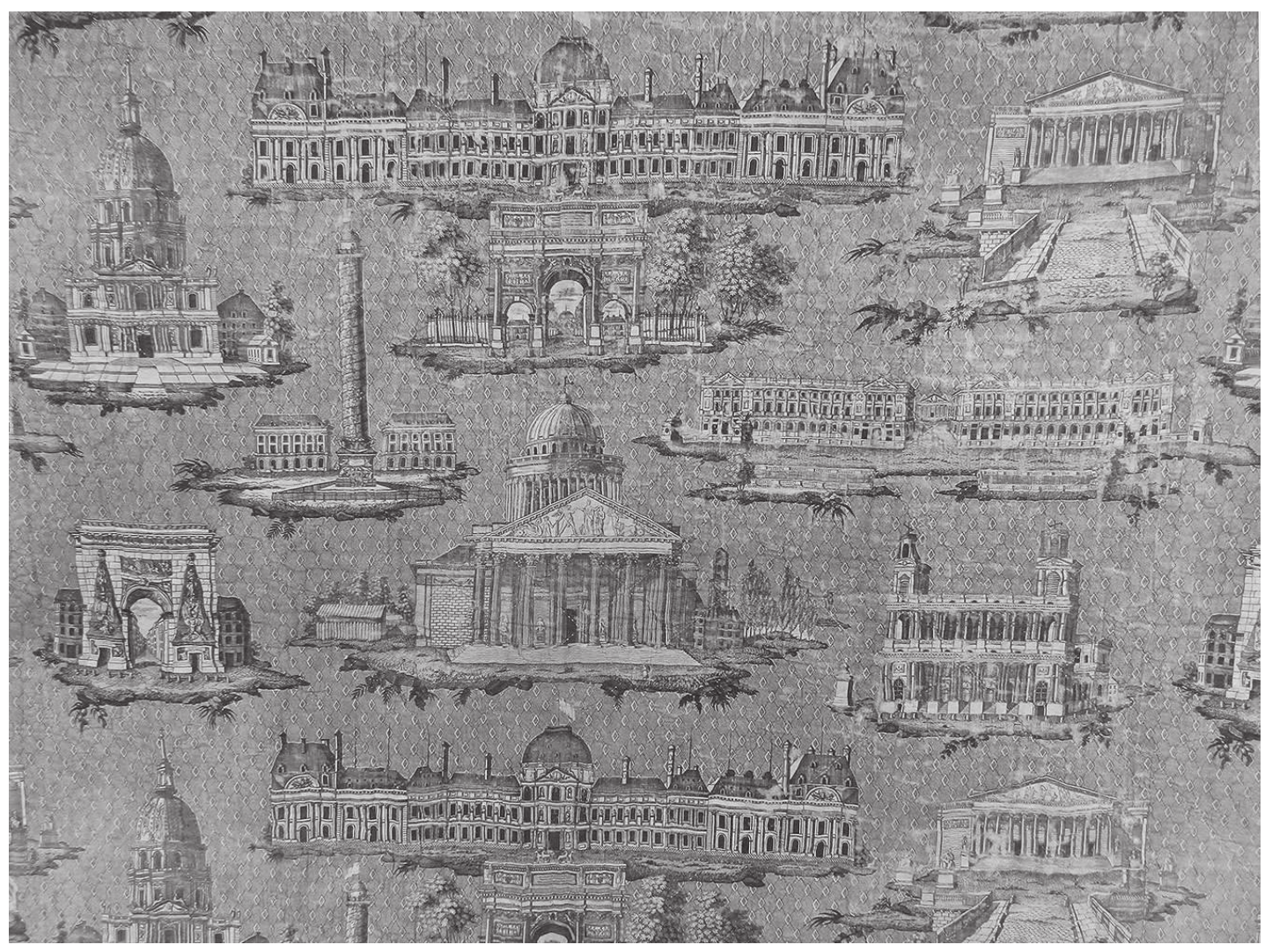

Ill. 6 : Les Monuments de Paris, manufacture Soëhnée l'Aîné \& C Cè Munster, vers 1816, impression à la plaque de cuivre, $80 \times 124 \mathrm{~cm}$, rapport $46 \times 80 \mathrm{~cm}$. MISE 976.182.1. (C) Cl. de l'auteur.

nade mais leur composition obéit à des impératifs d'usage dans les intérieurs qui expliquent ces différents choix. Le papier peint offre un panorama de la ville qui donne l'illusion d'être un visiteur alors qu'il a vocation à orner la totalité d'une pièce, ouvrant le mur vers l'extérieur. La toile imprimée est tendue sur le mur et recouvre le mobilier, lit, rideaux, fauteuils, permettant de percevoir sur des structures différentes plusieurs fois le même bâtiment. La perception du monument est complètement différente entre le papier peint en trompe l'œil où le propriétaire fait un voyage depuis son salon et l'étoffe qui évoque des souvenirs comme des estampes illustrant un récit de voyage.

Dans les années 1830, une manufacture rouennaise s'empare à nouveau du thème des monuments de Paris (ill. 7) dans une vision touristique où les monuments ne sont plus isolés comme des images iconiques, mais dans une succession de vues en perspective. La toile imprimée recherche les mêmes effets obtenus dans le papier peint en faisant du monument un véritable tableau. La vision pittoresque du panoramique est complétée par une idée de grandiose, alors que les monuments apparaissent disproportionnés à côté des minuscules habitants et touristes. La représentation de Paris est à nouveau incarnée par la colonne Vendôme, l'hôtel de Ville et le Palais Bourbon, des lieux déjà présents dans le papier peint. Alors que la toile de la manufacture d'Hartmann présente chaque monument isolé, dans cet exemple, 


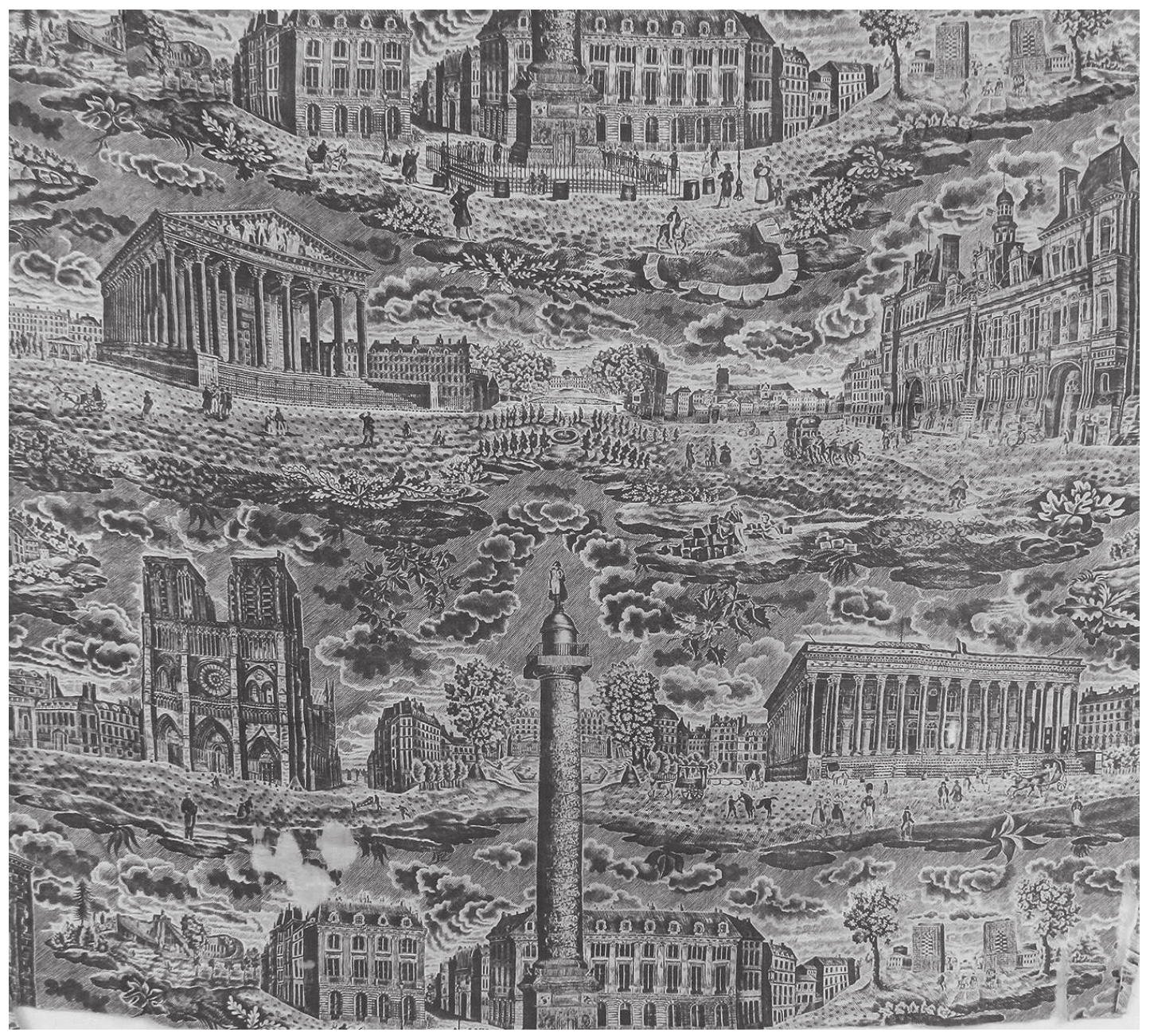

Ill. 7 : Les Monuments de Paris (Église de la Madeleine, Hôtel de Ville, parvis de Notre-Dame, Chambre des députés, colonne Vendôme), manufacture Rouen, années 1830, impression à la plaque de cuivre, $80 \times 124 \mathrm{~cm}$, rapport $56 \times 76 \mathrm{~cm}$. MISE 976.182.1. (C) Cl. de l'auteur.

l'environnement est pris en compte formant un véritable paysage et donnant une vision plus vivante de la ville, renforcée par la présence des personnages.

Le choix de la cathédrale Notre-Dame marque une évolution dans la perception des lieux à visiter dans la capitale alors que la génération romantique se passionne pour l'architecture gothique ${ }^{22}$. Depuis la fin du XVIII ${ }^{\mathrm{e}}$ siècle, la cathédrale est mentionnée dans les guides de Paris mais au même titre que bien d'autres monuments $^{23}$. La publication du roman de Victor Hugo Notre-Dame de Paris en 1831 lui donne une incroyable visibilité tandis que la représentation du monument cor-

22. Anne Coste, L'Architecture gothique, lectures et interprétation d'un modèle, Saint-Étienne, Publications de l'Université de Saint-Étienne, 1997, p. 79-80.

23. Luc-Vincent Thiéry, Paris tel qu'il étoit avant la Révolution, ou Description raisonnée de cette ville, de sa banlieue et de tout ce qu'elles contenoient de remarquable pour servir de guide aux amateurs et voyageurs français et étrangers, Paris, chez Delaplace, 1795-1796, t. 2, p. 80 ; Anonyme, Paris et ses curiosités; ou nouveau guide du voyageur à Paris, Paris, Chez Marchand, 1802, p. 86. 
respond à la découverte des édifices gothiques au moment où la Commission des monuments historiques se préoccupe de leur restauration ${ }^{24}$. La datation de la toile postérieure à 1833 , comme l'atteste la représentation de la colonne Vendôme surmonté de la sculpture d'Émile Serre représentant Napoléon en "Petit Caporal ", confirme l'évolution des monuments représentés et leur mise en scène.

Ces nombreuses compositions sur les monuments connus de la capitale proposent un nouveau décor de voyage alors que sous l'Empire, Paris s'est affirmée comme une capitale culturelle et une destination touristique ${ }^{25}$. Les représentations de Paris sont devenues sous la Restauration un thème décoratif très en vogue dans les arts industriels, le service d'assiettes Vues de Paris de la manufacture de Choisy atteste d'une diffusion dans des productions plus populaires où le monument est repris à des estampes avec leur légende ${ }^{26}$. Les architectures sont devenues des emblèmes de la ville, perçue à travers des sites emblématiques dont les différents choix sont révélateurs d'une évolution de l'intérêt du public, entre le Paris des bonapartistes et celui des royalistes. Entre les années 1810 et 1830, la perception de Paris passe en partie par les mêmes monuments mais les choix décoratifs mutent entre des symboles - monuments isolés chez Hartmann ou encadrés à Jouy - à des scènes pittoresques qui évoquent une succession d'estampes, illustrant un guide de voyage $^{27}$.

\section{Du décor au symbole, la valeur de l'architecture}

Le choix des monuments est fonction du thème proposé par le dessinateur ou initié par le fabricant, mais il est aussi révélateur d'une évolution de la perception de l'architecture qui d'un décor signifiant pour des scènes devient le sujet même de la composition. Entre les années 1800 et 1810, différents meubles créés à la manufacture de Jouy attestent de cette évolution, reprise dans les autres manufactures et adaptée au contexte politique. Le goût pour les références historiques

24. Anne Coste, L'Architecture gothique, op. cit., p. 69.

25. Alain Guyot et Chantal Massol (dir.), Voyager en France au temps du romantisme. Poétique, esthétique, idéologie, Grenoble, Ellug, 2003 ; Jean-Marie Goulemot, Paul Lidsky, Didier Masseau, Le Voyage en France, anthologie des voyageurs français et étrangers en France aux XIX $X^{e}$ et XX siècles, Paris, Robert Laffont, Bouquins, 1997.

26. Série de 12 assiettes "Vues de Paris ", fabriquées par la manufacture Paillart \& Hautin à Choisy entre 1824 et 1836 : Les Invalides, La Sainte-Chapelle et l'Hôtel de la Cour des Comptes, le Louvre, La Porte Saint-Denis, La Sorbonne, la colonne Vendôme et de la Colonne, La Madeleine, l'Hôtel des Monnaies, le pont Louis XVI (pont de la Concorde), autre vue de l'Hôtel des Monnaies, Porte Saint-Martin, vue du Pont du Jardin du Roi (pont d'Austerlitz).

27. Cette transformation de la représentation du monument n'est pas propre aux motifs imprimés sur toile, on la trouve dans les gravures d'illustrations où les monuments, isolés comme des œuvres d'art, apparaissent dans des scènes pittoresques, on peut citer l'ouvrage de Louis Bacler d'Albe, Promenades pittoresques et lithographiques dans Paris et ses environs, Paris, À la lithographie de G. Engelmann, 1822, même si le modèle ancien domine encore les guides: M. Lebrun, Manuel complet du voyageur dans Paris, ou nouveau guide de l'étranger dans cette capitale, soit pour la visiter, ou s'y établir, Paris, Robert Libraire, 1828. 


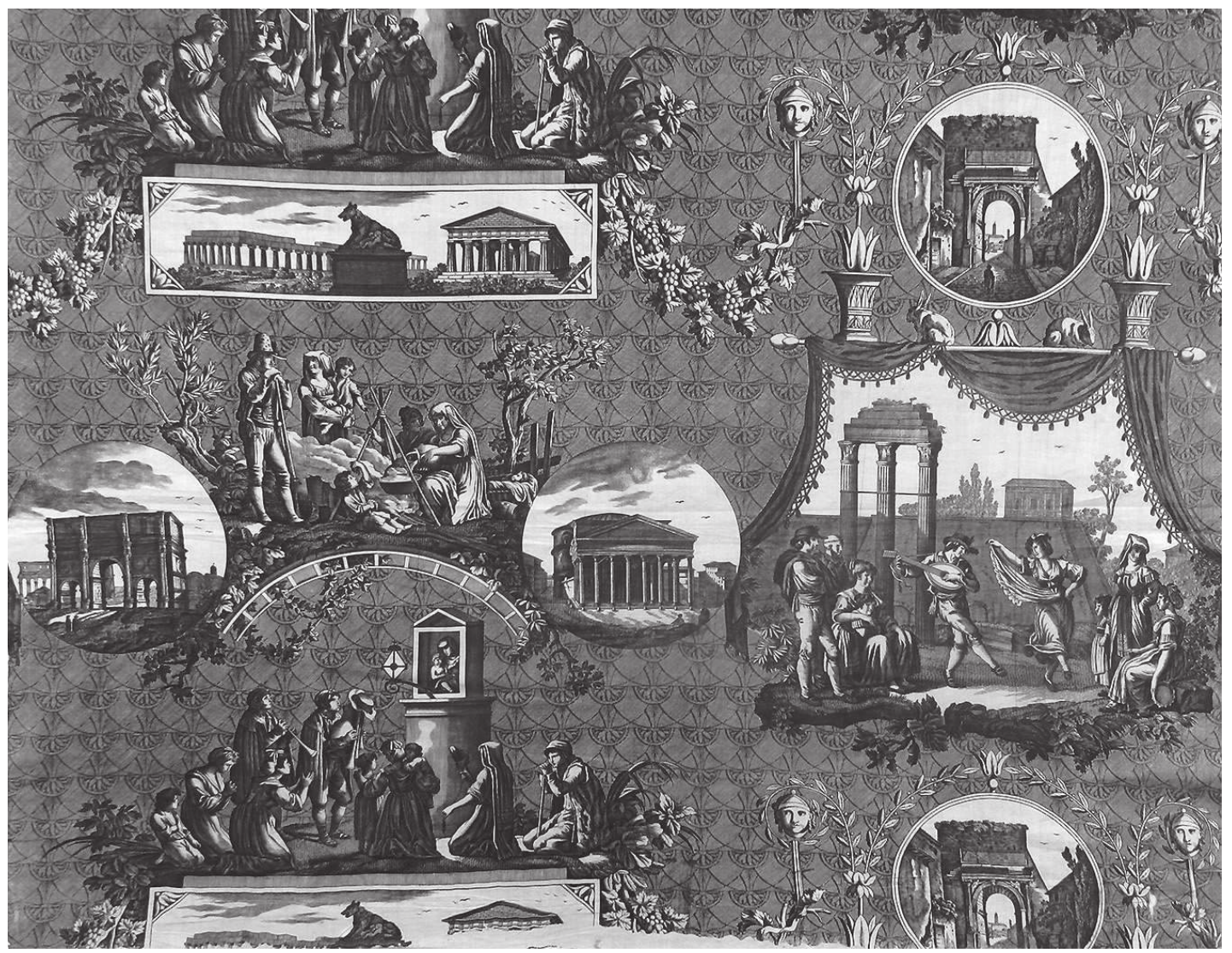

Ill. 8 : Le Romain, dessinateur Jean-Baptiste Huet, impression à la plaque de cuivre, 1811, D422, rapport 53,5 × $92 \mathrm{~cm}$. MISE inv.954.459.1(335). (C) Cl. de l'auteur.

conditionne la mise en scène des monuments tandis que le contexte politique renouvelle l'intérêt pour la portée symbolique du motif architectural.

En 1810, Jean-Baptiste Huet réalise pour la manufacture de Jouy une composition dans laquelle il décline des vestiges antiques célèbres d'Italie : l'arc de Constantin, celui de Drusus, le Panthéon, le Forum et le temple de Neptune à Paestum (ill. 8). Les motifs architecturaux contribuent à l'évocation d'un voyage comme ses précédentes compositions, les monuments étant accompagnés de personnages folkloriques repris aux gravures de Bartolomeo Pinelli ${ }^{28}$. Seule la place $\mathrm{du}$ Forum où se déroule une scène de danse reste dans la tradition des peintres de ruines avec des saynètes devant des vestiges antiques. Dans le reste du dessin, les scènes sont des motifs indépendants où les personnages apparaissent presque aussi grands que les architectures, donnant une impression de proximité et d'accessibilité aux monuments. Cette composition mêle plusieurs procédés décoratifs en isolant les monuments dans des médaillons, associés à des groupes de figures selon une ordonnance symétrique inspirée des compositions d'arabesque ${ }^{29}$. À partir de 1811,

28. Bartolomeo Pinelli, Raccolta di cinquanta costumi pittoreschi, Rome, Lorenzo Lazzari, 1809, 50 pl.

29. André Chastel, La Grotesque. Essai sur l'" ornement sans nom ", Paris, Le Promeneur, 1988 ; Bruno Pons, "Arabesques ou nouvelles grotesques", Alain Grüber (dir.), L'Art décoratif en Europe, Classique et Baroque, Paris, Citadelles et Mazenod, 1992, t. 2, p. 157-223 ; Alexia Lebeurre, "Les Loges 
la commercialisation de cette toile sous le titre "Le Romain " diffuse la mode pour les monuments antiques dans les étoffes. Leur représentation à travers un cadre donne l'illusion d'estampes disposées sur un mur, cette mise en scène renforce leur statut d'œuvres d'art provenant d'un passé prestigieux. En les assemblant à des scènes pittoresques, le peintre évoque aussi la ville comme une destination de voyage, culturel et pédagogique, symbole de la mémoire historique et artistique de l'Europe.

En 1818, la manufacture Zuber réalise le panoramique Vues d'Italie (ill. 9) dont les décors attestent d'une vision de l'Italie où les monuments sont omniprésents ${ }^{30}$. Le peintre Pierre-Antoine Mongin reprend d'autres gravures de Pinelli que celles utilisées dans la composition du Romain. L'artiste propose une vision pittoresque entre voyage et découverte du sublime où les architectures tiennent lieu de décor grandiose aux scènes et reprennent en partie l'image de l'Italie antique. Le livret publicitaire révèle les intentions du fabricant, les arguments privilégient une vision d'ensemble et la représentation de l'architecture se distingue peu des compositions du XVIII ${ }^{\mathrm{e}}$ siècle.

«Le paysage représente quelques-uns des sites et des monuments les plus remarquables de l'Italie ainsi que des scènes prises dans les habitudes et les mœurs des peuples qui habitent aujourd'hui les différentes provinces de cette belle contrée doit inspirer d'autant plus l'intérêt que toutes les vues et costumes sont fait d'après nature et que l'exécution aussi bien que le style de cette composition sont, sous tous les rapports, dignes de représenter des souvenirs de ce sol classique ${ }^{31}$."

On retrouve les vestiges du temple de Jupiter sur le Forum, déjà présents dans Le Romain tandis que le Colisée occupe une place centrale, d'autant plus importante que le monument est facilement identifiable par le consommateur. Avec ce panoramique, présenté à l'Exposition des produits de l'industrie de 1819, le fabricant propose un paysage pittoresque où l'architecture est un décor réaliste pour les habitants dans leurs costumes. Cette mise en scène évoque celle déployée par l'architecte Lebas dans la composition Les Monuments du midi, commercialisée par la manufacture de Jouy en 1818. La similitude esthétique entre la toile imprimée Les Monuments $d u$ midi et le panoramique Vues d'Italie est renforcée par une même scène empruntée à une gravure de Pinelli, transposée avec quelques variantes devant le Colisée de Rome dans le papier peint et les Arènes d'Arles dans le textile

de Raphaël, école moderne de l'arabesque ", sur quelques étapes de la réception d'un modèle, Thomas W. Gaehtgens, Christian Michel, Daniel Rabreau et Martin Schieder (dir.), L'Art et les normes sociales au XVIII siècle, Paris, Éditions de la Maison des sciences de l'homme, 2001, p. 425-441.

30. Bernard Jacqué, De la manufacture au mur, op. cit., p. 344.

31. "Paysage tableau en papier peint", texte publicitaire daté de 1818 de la manufacture Zuber à Rixheim, cité par Odile Nouvel-Kammerer (dir.), op. cit., nº 62, p. 300. 

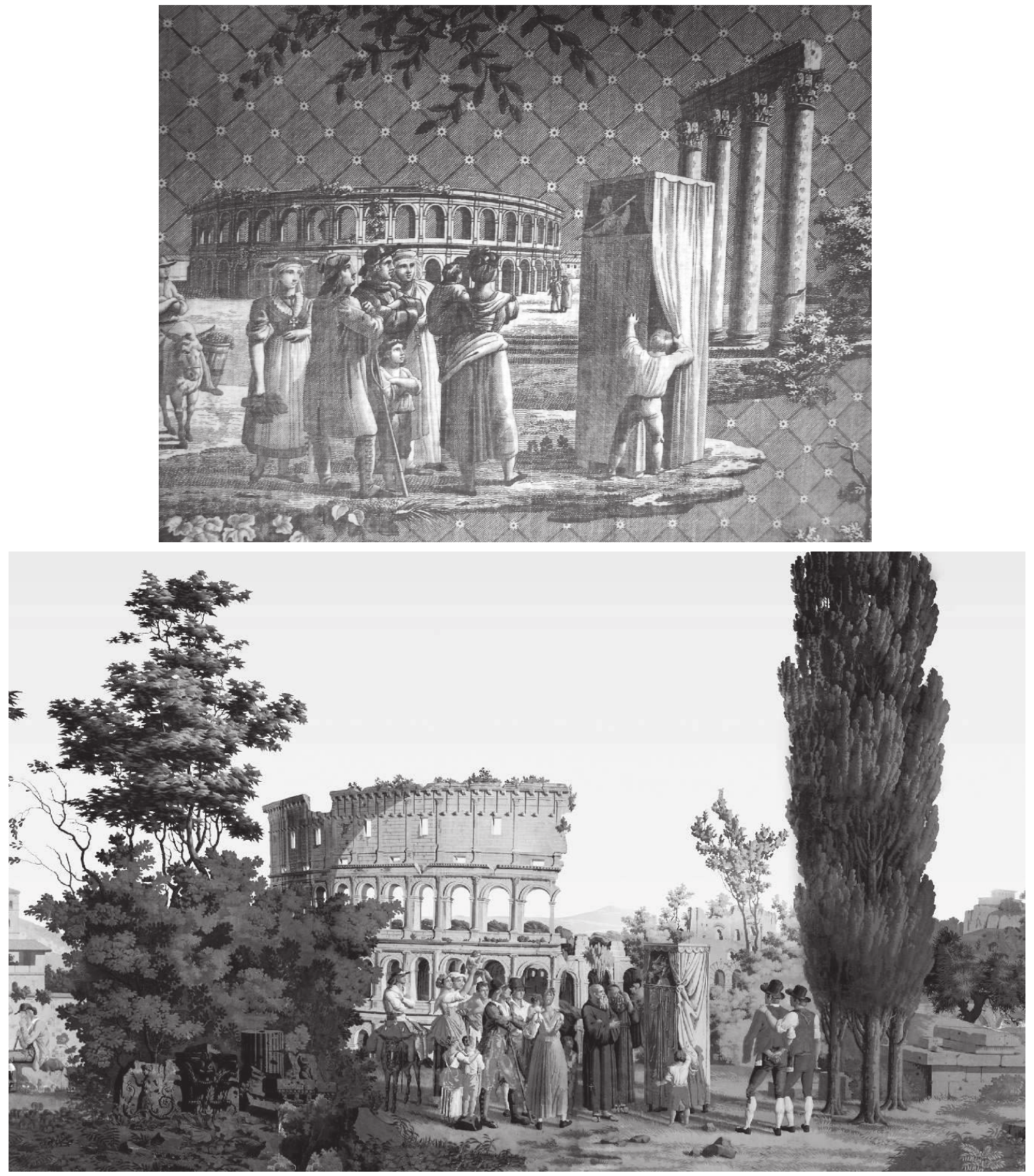

Ill. 9: en haut détail, Les Monuments $d u$ Midi, dessinateur Louis-Hippolyte Lebas, impression à la plaque de cuivre, 1818, D646, rapport $53 \times 94 \mathrm{~cm}$. MTJ inv.987.6.1. Cl. de l'auteur; en bas détail, Vues d'Italie, papier peint panoramique de la manufacture Zuber, Rixheim, 1818, dessinateur PierreAntoine Mongin, impression sur papier rabouté. (C) Avec l'aimable autorisation de l'entreprise Zuber. Toute reproduction interdite.

(ill. 9) ${ }^{32}$. Le panoramique copie fidèlement les figures dans leur attitude et leurs costumes, dans la toile imprimée, le nombre des personnages est réduit pour faciliter la lisibilité des motifs, mais Lebas rajoute deux personnages qui assistent à la scène à droite du guignol pour équilibrer l'ensemble.

32. Bartolomeo Pinelli, Raccolta di cinquanta costumi pittoreshi, op. cit., pl. 10, "ll casotto dei Burottini in Roma ". 


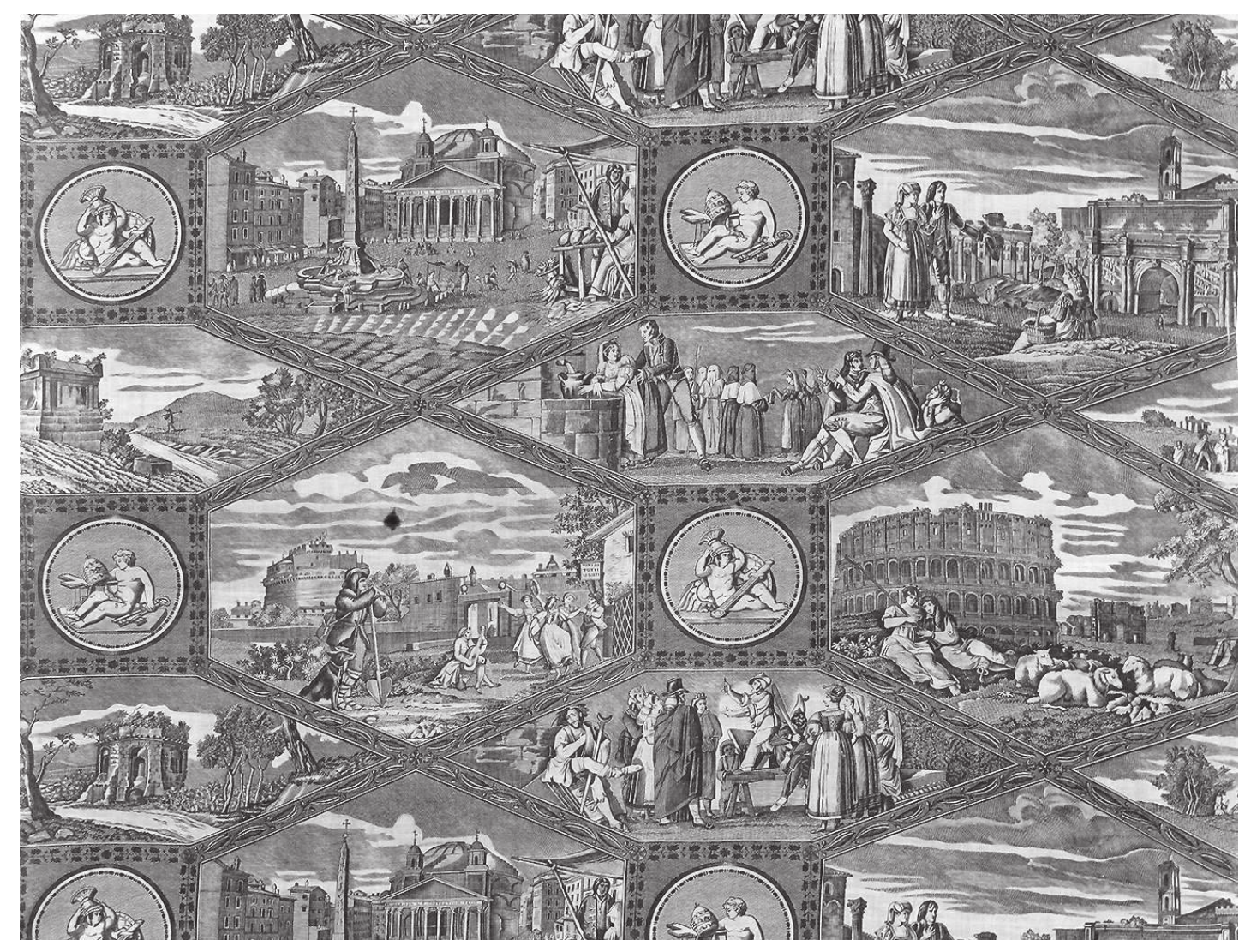

Ill. 10 : Les Monuments de Rome, manufacture rouennaise (signature Jamet), $1^{\mathrm{er}}$ quart du XIX $\mathrm{X}^{\mathrm{e}}$ siècle, impression à la plaque de cuivre, $230 \times 82 \mathrm{~cm}$, rapport $47 \times 81 \mathrm{~cm}$. MISE 976.252.1. (C) Cl. de l'auteur.

La toile imprimée n'a pu être à l'origine de la composition du peintre car il faut de long mois avant que la maquette du panoramique soit imprimée sur papier, plus qu'une simple coïncidence, ces compositions si proches attestent d'un goût généralisé pour les représentations d'architecture associées aux scènes de genre. D'autres compositions de meubles reprennent cette formule pour représenter des monuments. Dans le deuxième quart du XIX $\mathrm{X}^{\mathrm{e}}$ siècle, les manufactures rouennaises déclinent à leur tour ce thème pour proposer de nouveaux meubles à personnages. Le graveur Jamet réalise une composition dont l'organisation en mosaïque tranche dans le corpus des toiles normandes (ill. 10). Des médaillons inscrits dans des carrés reprennent des motifs à l'antique intercalés avec des formes hexagonales qui encadrent des vues de Rome. L'inspiration des vedute et le pittoresque des saynètes folkloriques du graveur Pinelli sont encore très présents, mais les monuments ne sont plus réduits à des vignettes comme dans Le Romain. Si l'intérêt pour l'architecture antique prime - en témoignent les scènes avec le Colisée ou l'obélisque devant le Panthéon - la composition dépasse la simple représentation des monuments de l'Italie pour devenir la mise en scène de la ville éternelle. Le graveur choisit des motifs qui incarnent Rome pour tous les consommateurs, des monuments antiques au château Saint-Ange tandis que les personnages en habits contemporains incarnent les différentes composantes de la société italienne. 
Les motifs d'architecture sont déclinés par les dessinateurs pour évoquer des lieux emblématiques, des périodes historiques donnant lieu parfois à une mise en scène du politique. Sous la Révolution, Oberkampf, contraint d'actualiser un dessin mettant en scène Louis XVI, fait insérer dans un médaillon la forteresse de la Bastille en cours de démolition d'après le tableau d'Hubert Robert ${ }^{33}$. Le motif est alors choisi comme symbole politique et non pour sa valeur architecturale, mais il est révélateur du pouvoir de représentation que le monument peut induire dans une composition pour décorer les intérieurs ${ }^{34}$. Dans la composition du graveur Louis Henri Bréviaire sur l'Égypte, les monuments sont disposés autour de la figure centrale, le sultan Muhammad Ali Pacha vice-roi d'Égypte d'après un portrait équestre d'Horace Vernet, diffusé par une lithographie datée de $1818^{35}$. La scène principale de la composition est le personnage incarnant le pouvoir politique, les architectures ne sont que des motifs secondaires qui l'accompagnent.

Dans ses compositions, l'architecte Lebas imagine un programme iconographique où l'art se mêle au politique, en 1816 avec Les Monuments de Paris, il propose une évocation des réalisations architecturales, rattachées aux plus illustres rois de France. Ce dessin est réalisé alors qu'il commence la construction de la chapelle Expiatoire à la mémoire de Louis XVI et Marie-Antoinette ${ }^{36}$. Les quatre ensembles architecturaux qui forment la composition, doivent être appréhendés sous l'angle d'un discours propre à séduire une nouvelle clientèle, sensible à l'histoire de la monarchie française. Le Pont-Neuf avec la statue en bronze d'Henri IV qu'il réhabilite avant même sa réinstallation, la Fontaine des Innocents, le Panthéon ou plutôt Sainte-Geneviève rendue au culte en 1806 par Napoléon, enfin le Louvre avec la colonnade de Perrault sont inscrits dans des médaillons. Chaque élément est plus ou moins décoré, surmonté d'un portrait en médaille du roi de France correspondant au monument : Henri IV, Henri II, Louis XVI et Louis XIV. Lebas flatte le désir de reconnaissance de l'autorité royale, mais ses choix iconographiques

33. Aziza Gril-Mariotte, «Christophe-Philippe Oberkampf (1738-1815) et l'industrie des toiles peintes en France. L'impact du protestantisme sur son parcours et la création ", Revue d'histoire du protestantisme, avril-mai-juin 2016, p. 207-227: Louis XVI, restaurateur de la liberté, dessinateur Jean-Baptiste Huet, impression à la plaque de cuivre, 1789, numéro de dessin 140, rapport $100 \times 92 \mathrm{~cm}$.

34. Pour continuer à vendre ce meuble, Oberkampf le rebaptise «le dessin de la Révolution », mais la Terreur l'oblige à liquider ses dernières pièces. Malgré une vente limitée dans le temps, il existe de très nombreux exemplaires de ce motif, preuve d'un véritable succès commercial, le musée de la toile de Jouy et le musée de l'Impression sur étoffes de Mulhouse possèdent plusieurs exemplaires ainsi que le Cooper Hewitt Museum de New York.

35. Mylène Doré (dir.), Quand les toiles racontent des histoires, les toiles d'ameublement normandes au XIX $X^{e}$ siècle, Musée des Traditions et Arts Normands, château de Martainville, du 12 mai 2006 au 7 janvier 2008, p. 171.

36. Vassiliki Petridou, "La doctrine de l'imitation dans l'architecture française dans la première moitié du XIX ${ }^{\mathrm{e}}$ siècle, du néo-classicisme au romantisme à travers l'œuvre de Louis-Hippolyte Lebas (1782-1867) ", thèse de doctorat d'histoire de l'art, sous la direction du Professeur Bruno Foucart, Université Paris-IV, 1992, p. 396 : Les Monuments de Paris, dessinateur Louis-Hippolyte Lebas, impression à la plaque de cuivre, 1816, numéro de dessin 600, rapport $52,5 \times 82 \mathrm{~cm}$. 
ne sont pas seulement idéologiques, à travers ces monuments, il rend hommage aux grands architectes français, depuis Baptiste Androuet du Cerceau jusqu'à JacquesGermain Soufflot en passant par Claude Perrault et Pierre Lescot. En associant le règne d'un roi de France à un architecte, il propose une vision historiographique de l'histoire monumentale de Paris. L'attention particulière qu'il accorde au cadre de la colonnade du Louvre, complété par des allégories (géographie, peinture et sculpture) se réfère explicitement à la création des académies sous le règne de Louis XIV. La représentation des monuments prend un caractère emblématique et commémoratif, la toile pour ameublement devient le support d'une histoire politique et artistique de la capitale et au-delà de la nation française.

Ce lien entre l'architecture et l'affermissement d'un passé national n'est pas nouveau mais dans le contexte de la défaite de l'Empereur et de l'occupation de Paris par les Alliés, la représentation de la capitale prend un aspect politique, à tel point que le panoramique de Dufour est modifié après 1815 en supprimant la sculpture de Napoléon au sommet de la colonne Vendôme ${ }^{37}$. L'attrait de Paris et la mode des panoramiques dans les régions germaniques n'empêchent pas la commercialisation de la première version comme l'atteste plusieurs exemples encore en place. Dès 1812, le panoramique est posé dans une des pièces des appartements de parade du Prince Nassau-Weilberg dans le château Schloß à Weilburg an der Lahn, entre Bonn et Francfort tandis que le Schloß Herrnsheim près de Worms l'utilise pour le décor d'un petit salon des appartements privés ${ }^{38}$.

Le meuble sur les monuments de Paris (ill. 6), imprimé par la manufacture d'Hartmann vers 1816, propose, au-delà d'une vision touristique, un véritable programme iconographique en faveur de l'Empire et à la mémoire de Napoléon. La composition accumule des monuments dédiés à la Grande Armée : l'Arc de triomphe du Carroussel et la colonne Vendôme, les Invalides dont le dôme est sous l'Empire le "Panthéon des gloires militaires de la France ", bien avant le rapatriement des cendres de l'Empereur tandis que la perspective de la place de la Concorde permet d'apercevoir la Madeleine, consacrée en 1807, le temple « à la gloire des Armées françaises " ${ }^{39}$. Les autres bâtiments qui complètent le dessin évoquent la politique de l'Empire et la présence de Napoléon à Paris : le Palais des Tuileries, résidence impériale, le Palais Bourbon avec la colonnade de l'architecte Bernard Poyet dont on devine sur le fronton le bas-relief à la gloire de l'Empereur, ainsi que l'église Saint-Sulpice dont la place est achevée en 1811. Chaque lieu rappelle avec plus ou moins d'évidence Napoléon et répond à la nostalgie de l'Empire,

37. En France, la " Maison Roux » située à Dieulefit présente une version postérieure à 1815 sur les murs de la salle à manger dont l'installation est datée de 1819.

38. Bernard Jacqué et Georgette Pastiaux-Thiriat (dir.), Joseph Dufour, manufacturier du papier peint, Rennes, Presses universitaires de Rennes, p. 202-204.

39. Thierry Sarmant (dir.), Napoléon et Paris : rêves d'une capitale, catalogue de l'exposition du musée Carnavalet, du 8 avril au 30 août 2015, Paris-Musées, 2015. 
en accord avec les idées politiques du fabricant ${ }^{40}$. L'étude de la maquette conforte l'idée que les monuments de Paris sont associés au retour de l'Empereur, mais les événements politiques obligent le fabricant à modifier plusieurs détails ${ }^{41}$. Dans la toile, la colonne Vendôme apparaît sans la sculpture de Napoléon, pourtant présente sur le dessin tandis que le drapeau sur le Panthéon est remplacé par une croix bien que Napoléon ait rendue l'église Sainte-Geneviève au culte en 1806 et le drapeau sur le Palais des Tuileries, tricolore dans le dessin est devenu blanc lors de l'impression sur toile. Dans cet assortiment de monuments, la porte Saint-Denis avec l'arc de triomphe de François Blondel célébrant la traversée du Rhin de Louis XIV pendant la guerre de Hollande, dénote. Le monument n'a pas été rajouté pour tenir compte de l'avènement de la Restauration car il est déjà présent dans le dessin préparatoire. Le fabricant est aussi intervenu dans l'appréhension $\mathrm{du}$ monument, alors que le dessin donne une vision pittoresque de la capitale peuplée de personnages découvrant les bâtiments, dans la toile imprimée, les monuments apparaissent isolés de la ville tels des emblèmes.

Ces détails montrent comment l'idée d'un meuble sur le Paris sous l'Empire évolue vers une vision historique de la ville et préfigure "la légende napoléonienne ", entretenue par les Bonapartistes sous la Restauration dont les exemples dans le textile sont nombreux ${ }^{42}$. Cette appréhension du monument parisien perdure, comme l'atteste l'exemplaire imprimé à Rouen dans les années 1830 où la colonne Vendôme est surmontée de la sculpture de Napoléon en "petit caporal» réalisée par la Monarchie de Juillet (ill. 7). La représentation de la cathédrale Notre-Dame peut aussi être perçue par les consommateurs comme une référence au règne de Napoléon alors que l'église a été le théâtre de son couronnement. Le contexte historique apparaît favorable à une représentation des monuments de Paris, associée aux idéaux politiques et à la mise en scène d'un passé historique, plus ou moins récent.

La représentation de l'architecture est une des facettes du goût pour le passé qui prédomine la création des arts au XIX ${ }^{\mathrm{e}}$ siècle. Lorsque ces motifs se propagent dans les meubles à personnages, ils varient du décor signifiant pour des saynètes jusqu'à devenir le thème de la composition et parfois les seuls motifs du dessin.

40. Le journal d'Henri Lebert est truffé de références à l'Empire, de l'admiration envers l'Empereur et du regret de sa chute, sentiments politiques qu'il les partage avec son patron : op. cit., vol. II (18141817), p. 10. Il s'exprime en ces termes en mai 1814 : "Ainsi, cette puissante couronne impériale, on l'a brisa pour la jeter sur une île! Elle a répandu trop d'éclat sur notre patrie, aujourd'hui blanche et décolorée [...]. Le drapeau tricolore, nos aiglons victorieux, notre gloire, tout cela a dû disparaître devant des vaincus de la veille ». Sans surprise, le retour de l'Empereur est accueilli avec enthousiasme, il est probable que le dessin date de ce moment, mais Henri Lebert n'y fait pas allusion.

41. Le dessin est conservé au Cooper Hewitt Museum https://collection.cooperhewitt.org/objects/ 18102811/ (URL consulté le 20/10/2016).

42. La manufacture de Jouy commercialise en 1819 un " meuble militaire » mettant en scène la Grande Armée, préfigurant l'engouement pour les motifs napoléoniens dans les impressions normandes, voir Aziza Gril-Mariotte, Les Toiles de Jouy, op. cit., p. 219 : "dessin militaire", dessinateur Horace Vernet, impression à la plaque de cuivre, 1819, Numéro de dessin 580 , rapport $52,5 \times 89 \mathrm{~cm}$. 
Les toiles représentant des monuments de Paris attestent de cette évolution. La composition de Lebas est vendue par la manufacture de Jouy sous le titre commercial "Les Monuments de Paris", revendiquant ainsi la mise en scène décorative des architectures emblématiques de la ville. Le succès que rencontre ce meuble encourage l'artiste à réaliser la composition, vendue en 1818 sous le titre "Les Monuments du Midi ", où les motifs d'architecture occupent de manière majestueuse un paysage grandiose pour des saynètes à nouveau empruntées à d'autres gravures de Pinelli ${ }^{43}$. Lebas réunit les monuments antiques du sud de la France les plus connus, la Maison carrée de Nîmes, l'arc de triomphe d'Orange, les arènes d'Arles, les colonnes de Riez et le Pont flavien de Saint-Chamas, inspirés du Voyage dans le midi de la France de Millin avec des personnages contemporains, évoquant une histoire nationale toujours vivante ${ }^{44}$. L'architecture et les personnages sont conçus comme un ensemble cohérent, les scènes de genre mettent en valeur les monuments en renforçant leur perception sur le fond de la toile.

Lebas reprend l'idée du "voyage pittoresque " diffusée dès la fin du XVIII ${ }^{\mathrm{e}}$ siècle et déjà introduit dans les meubles à personnages par Jean-Baptiste Huet avec Le Romain $^{45}$. La toile s'apparente à un récit de voyage illustré où le pittoresque du paysage se mêle aux vestiges antiques, à la nature et au folklore des costumes, avant même la publication des célèbres Voyages pittoresques et romantiques de l'ancienne France par le baron Taylor et Charles Nodier ${ }^{46}$. Les monuments antiques du Midi de la France sont depuis longtemps des lieux de passage du Grand tour et un détour obligé pour les artistes se rendant en Italie ${ }^{47}$. Malgré ses voyages en Italie entre 1804 et $1811^{48}$, Lebas se contente de puiser dans les recueils de gravures

43. Bartolomeo Pinelli, Raccolta di cinquanta costumi pittoreschi, op. cit., la Maison Carrée de Nîmes, $\mathrm{n}^{\circ}$ 4, «le mozzatore ritarnando dopo la vendemmia in Roma ", La Danse; l'arc de Triomphe d'Orange, $\mathrm{n}^{\circ}$ 7, "Ballo del Orso ", la Danse de l'Ours ; Les arènes d'Arles et les colonnes de Riez, $\mathrm{n}^{\mathrm{o}} 10$ "II casotto dei Burottini in Roma », le Guignol ; Le Pont Flavien de Saint-Chanas, ${ }^{\circ}$ 12, "II gioco di boccia in Roma ", le jeu de boules.

44. Aubin-Louis Millin, Voyage dans le midi de la France, 1807-1811, pl. LXXII et LXXII.

45. Jean-Benjamin de La Borde, Description générale et particulière de la France, ouvrage enrichi d'estampes d'après les dessins des plus célèbres artistes, Paris, 1781-1784 et le Voyage pittoresque de la France avec la description de toutes les provinces, Paris, 1784-1789, Nouveau voyage pittoresque de la France orné de 360 gravures exécutées par les meilleurs artistes de Paris sur des dessins fait d'après nature avec la plus grande fidélité et représentant des vues des principales villes de France, ports de mer, monuments anciens et modernes, Paris, Ostervald l'aîné, 1817.

46. James Taylor, Charles Nodier, Alphonse de Cailleux, Voyages pittoresques et romantiques de l'ancienne France, Paris, éd. Didot, 1820-1878.

47. Michel Vovelle, "La découverte de la Provence, ou les primitifs de l'ethnographie provençale, 1750-1850", De la cave au grenier, Québec, S. Fleury, 1980, p. 407-435; Nicolas Bourguinat (dir.), L'Invention des Midis : représentations de l'Europe du Sud, XVIII $-X X^{e}$ siècle, acte du colloque "Meridionalité et insularité : l'invention d'une Europe du Sud, XVIII"-XX ${ }^{\mathrm{e}}$ siècle ", 2011, Université de Strasbourg, Presses universitaires de Strasbourg, 2015.

48. Vassiliki Petridou, op. cit., p. 395, il effectue trois voyages en Italie, en 1804, de 1806 à 1808 et en 1811. L'artiste a pu aussi réemployer ses croquis réalisés lors de ses voyages mais la consultation des dessins conservés à la Bibliothèque de l'Institut de France n'a pas permis de vérifier une telle hypothèse. 
disponibles pour assembler des vues des monuments antiques avec des scènes folkloriques dans une vision qui s'inscrit dans l'esthétique du paysage de ruines au XVIII ${ }^{\mathrm{e}}$ siècle.

Malgré des compositions très différentes, les deux dessins de meubles à personnages réalisés par Lebas représentent une sélection de motifs évoquant un passé glorieux qui confere à l'architecture une valeur de monuments historiques ${ }^{49}$. L'utilisation du terme "monuments " dans le titre commercial de ces étoffes révèle une attention nouvelle dans les arts décoratifs à la représentation de l'architecture et à son appréhension par les consommateurs. Sans doute parce qu'il est avant tout un architecte plutôt qu'un dessinateur, Lebas, tout en rejoignant l'idée que la mise en scène du monument dans les intérieurs est associée au souvenir d'un voyage entrepris ou rêvé, accorde un pouvoir d'attraction particulier aux monuments représentés, mettant en scène un patrimoine national.

$\mathrm{Au} \mathrm{XIX}^{\mathrm{e}}$ siècle, la représentation de l'architecture contribue au renouvellement des thèmes pour les manufactures de toiles peintes et de papiers peints, au même titre que le goût pour la littérature, les motifs antiques ou encore les sujets historiques. Pourtant en diffusant ces motifs, les industriels ne font que s'approprier une esthétique des monuments déployée dans la peinture depuis les années 1770. Du reste, à la manufacture de Jouy, ces décors ne résultent pas de l'intervention de nouveaux dessinateurs, les premières compositions avec des monuments sont réalisées par le peintre Jean-Baptiste Huet (1745-1811). Ses dessins reflètent l'intérêt d'un large public pour les architectures anciennes et lancent une mode durable dans les meubles à personnages. L'artiste s'approprie une mise en scène des motifs architecturaux en leur accordant une valeur artistique et historique qui sera reprise par ses successeurs à Jouy et dans d'autres manufactures. Les représentations de monuments prestigieux donnent une dimension emblématique à la représentation de l'architecture alors que les dessinateurs choisissent des modèles évoquant un passé glorieux. Ce goût pour l'histoire monumentale est visible dans les toiles consacrées aux monuments antiques, incarnant l'idéal classique dont les images sont diffusées depuis longtemps. Les dessinateurs ne donnent pas une vision érudite de l'Antiquité, ils sélectionnent les vestiges les plus connus pour séduire les consommateurs. En choisissant des monuments antiques, puis les réalisations de grands architectes, ils accompagnent cette vision historique d'une représentation d'œuvres d'art. Les motifs sont situés dans un espace géographique, mais ils incarnent aussi des modèles artistiques, considérés comme des emblèmes de l'art. La représentation des monuments prend parfois une dimension allégorique, accentuée par les décors qui les accompagnent ou un traitement décoratif particulier. Les changements dans la perception du monument reflètent les nouvelles préoccupations de l'architecture. L'époque hérite de la Révolution française la notion de monuments comme biens appartenant à la nation, cet héritage concourt à développer la notion

49. Alain Schnapp, "Robert des ruines" le peintre face aux monuments antiques ", Guillaume Faroult (dir.), Hubert Robert (1733-1808) un peintre visionnaire, catalogue de l'exposition au musée du Louvre, du 8 mars au 30 mai 2016, Paris, Somogy, p. 84-93. 
d'intérêt public et collectif en faveur des bâtiments anciens. De nombreuses publications ont contribué à diffuser ces idées, apparues dès la seconde moitié du $\mathrm{XVIII}^{\mathrm{e}}$ siècle chez les érudits. Les gravures des monuments antiques de la Gaule ont fait connaître un passé national, longtemps occulté par les modèles italiens. Dans les toiles comme dans les panoramiques, la mise en scène de l'architecture, à part quelques propositions originales, reste dépendante des modèles qui circulent et de la référence au paysage en peinture où le pittoresque prime.

La différence entre le textile imprimé et le papier peint réside dans la composition des dessins qui prend en compte des usages différents dans les intérieurs. Chaque support déploie sa propre conception décorative : les étoffes, avec leur fond orné sur lequel se détachent les motifs affirment la matérialité du mur ; à l'inverse, le panoramique fait du papier peint un trompe-l'œil qui ouvre la pièce vers l'extérieur. Ces compositions antinomiques résultent de contraintes techniques et esthétiques propres à chacune de ces productions. Dans le textile, la maquette gravée sur une plaque de cuivre imprime le dessin dans la largeur d'un lé de toile autant de fois que nécessaire. Les saynètes et la multiplication des formes décoratives limitent la perception de la répétition du dessin une fois l'étoffe utilisée dans l'ameublement. Le panoramique est composé d'une série de lés de papier, une fois en place dans les intérieurs, les motifs imprimés à la détrempe avec des planches de bois créent un décor illusionniste qui imite la peinture. Le panoramique est formé de plusieurs scènes qui se raccordent en un seul paysage, mais qui peuvent aussi être utilisées comme des tableaux indépendants pour chaque mur. En fonction de la taille des pièces, le papier peint est complété par des motifs en trompe l'œil de bordures pour un usage en tableau ou de balustrades et de colonnes pour donner l'illusion d'un paysage ${ }^{50}$. Les toiles imprimées servent à fabriquer des lits, à revêtir des fauteuils pour l'ameublement d'une chambre. Les médaillons et les différentes saynètes sont ainsi distribués par le tapissier pour former des ensembles agréables à la vue, sans que leur répétition ne gêne la perception des motifs ${ }^{51}$.

Dans les étoffes d'ameublement et les panoramiques, la place de l'architecture reflète une nouvelle vision des monuments et l'intérêt du public pour le patrimoine monumental. Les compositions mettant en scène des monuments isolés restent peu nombreuses mais leur commercialisation par plusieurs manufactures suggère l'engouement des consommateurs pour ces modèles. Les fabricants privilégient la diversité des sujets décoratifs, hors la manufacture de Jouy impriment à quelques années d'intervalle des compositions sur ce thème qui semble plaire particulièrement aux consommateurs. Les exemples de représentation d'architecture dans les arts industriels, comme la faïence, sont une preuve supplémentaire de leur succès

50. Voir les reproductions d'exemples en place: Sabine Thümmler, "Installations historiques de papiers peints panoramiques de Joseph Dufour en Allemagne ", Bernard Jacqué et Georgette Pastiaux-Thiriat (dir.), Joseph Dufour, op. cit., p. 202-203 pour les Monuments de Paris et p. 206 pour Vues d'Italie.

51. Aziza Gril-Mariotte, "Indiennes, toiles peintes et toiles de Jouy, de nouvelles étoffes d'ameublement au XVIII ${ }^{\mathrm{e}}$ siècle ", Histoire de l'Art, Paysages urbains, en varia n ${ }^{\circ} 65$, octobre 2009, p. 143-154. 
auprès d'un large public que ces motifs servent de décor à un autre sujet ou bien qu'ils forment le thème du dessin ${ }^{52}$. En général, les monuments personnifient des villes, des personnages historiques et leur fortune iconographique est révélatrice $\mathrm{du}$ goût pour l'histoire. Ils sont choisis parce qu'ils sont liés à l'image d'un pays et d'une culture, la place de l'architecture dans les compositions fait du paysage un lieu d'histoire dont le but est de faire voyager et rêver le consommateur qu'il soit devant le panoramique de son salon ou dans son lit.

Aziza GRIL-MARIOTTE Université de Haute-Alsace

52. Les manufactures rouennaises qui se spécialisent dans ce genre d'impressions dans les années 1830 et 1840 ont très souvent recours à des motifs d'architectures dans les toiles à sujets bibliques ou dans les scènes historiques, voir Mylène Doré (dir.), Quand les toiles racontent des histoires, les toiles d'ameublement normandes au XIX siècle, catalogue d'exposition, musée des Traditions et Arts normands, château de Martainville, du 12 mai 2006 au 7 janvier 2008, Rouen, éd. des Falaises, 2007. 